\title{
New Differential Operator for Holomorphic Functions
}

\author{
Abbas Kareem Wanas \\ Department of Mathematics, College of Science, University of Al-Qadisiyah, Iraq \\ e-mail: abbas.kareem.w@qu.edu.iq
}

\begin{abstract}
In this paper, by making use of binomial series, we define a new differential operator of holomorphic functions in the open unit disk. Also, we introduce and investigate two new classes containing this new operator associated with differential subordinations and superordinations. Furthermore, we determine important properties for functions belonging to these classes.
\end{abstract}

\section{Introduction}

Indicate by $\mathcal{H}(\mathbb{D})$ the collection of holomorphic functions in the unit disk $\mathbb{D}=$ $\{z \in \mathbb{C}:|z|<1\}$. For $n \in \mathbb{N}$ and $a \in \mathbb{C}$, let $\mathcal{H}[a, n]=\left\{f \in \mathcal{H}(\mathbb{D}): f(z)=a+a_{n} z^{n}\right.$ $\left.+a_{n+1} z^{n+1}+\cdots\right\}$.

Let we name by the notation $\mathcal{A}$ the collection of all holomorphic functions in $\mathbb{D}$ and having the form:

$$
f(z)=z+\sum_{n=2}^{\infty} a_{n} z^{n}, \quad z \in \mathbb{D} .
$$

"With a view to recalling the principal of subordination between holomorphic

Received: August 30, 2019; Accepted: September 28, 2019

2010 Mathematics Subject Classification: 30C45, 30C80.

Keywords and phrases: holomorphic function, convex function, differential subordination, differential superordination, differential operator.

Copyright (C) 2019 Abbas Kareem Wanas. This is an open access article distributed under the Creative Commons Attribution License, which permits unrestricted use, distribution, and reproduction in any medium, provided the original work is properly cited. 
functions, let the functions $f$ and $g$ be holomorphic in $\mathbb{D}$, we say that the function $f$ is subordinate to $g$, if there exists a Schwarz function $w$ holomorphic in $\mathbb{D}$ with $w(0)=0$ and $|w(z)|<1(z \in \mathbb{D})$ such that $f(z)=g(w(z))$. This subordination is indicated by $f \prec g$ or $f(z) \prec g(z)(z \in \mathbb{D})$. Furthermore, if the function $g$ is univalent in $\mathbb{D}$, then we have the following equivalent (see [4]), $f(z) \prec g(z) \Leftrightarrow f(0)=g(0)$ and $f(\mathbb{D}) \subset g(\mathbb{D}) . "$

Definition 1.1 [6]. Let $p, h \in \mathcal{H}(\mathbb{D})$ and $\psi(r, s ; z): \mathbb{C}^{2} \times \mathbb{D} \rightarrow \mathbb{C}$. If $p$ and $\psi\left(p(z), z p^{\prime}(z) ; z\right)$ are univalent functions in $\mathbb{D}$ and if $p$ satisfies the first-order differential superordination

$$
h(z) \prec \psi\left(p(z), z p^{\prime}(z) ; z\right),
$$

then $p$ is called a solution of the differential superordination (1.2). (If $f$ is subordinate to $g$, then $g$ is superordinate to $f$ ). An analytic function $q$ is called a subordinate of (1.2), if $q \prec p$ for all $p$ satisfying (1.2). A univalent subordinate $\tilde{q}$ that satisfies $q \prec \tilde{q}$ for all the subordinants $q$ of (1.2) is called the best subordinant.

Now, by making use of the binomial series

$$
(1-\alpha)^{k}=\sum_{m=0}^{k}\left(\begin{array}{l}
k \\
m
\end{array}\right)(-1)^{m} \alpha^{m}, \quad\left(k \in \mathbb{N}=\{1,2, \ldots\}, m \in \mathbb{N}_{0}=\mathbb{N} \cup\{0\}\right)
$$

and for $f \in \mathcal{A}, \quad \alpha \in \mathbb{R}, \quad \beta \geq 0$ with $\alpha+\beta>0$ and $\delta \in \mathbb{N}_{0}=\mathbb{N} \bigcup\{0\}$, we define the differential operator $W_{\alpha, \beta}^{k, \delta}: \mathcal{A} \rightarrow \mathcal{A}$ as follows:

$$
\begin{aligned}
& W_{\alpha, \beta}^{k, 0} f(z)=f(z), \\
& W_{\alpha, \beta}^{k, 1} f(z)=\frac{\left(1-(1-\alpha)^{k}\right) f(z)+\left(1-(1-\beta)^{k}\right) z f^{\prime}(z)}{2-(1-\alpha)^{k}-(1-\beta)^{k}}=W_{\alpha, \beta}^{k} f(z) \\
& \vdots \\
& W_{\alpha, \beta}^{k, \delta} f(z)=W_{\alpha, \beta}^{k}\left(W_{\alpha, \beta}^{k, \delta-1} f(z)\right) .
\end{aligned}
$$

If $f$ is given by (1.1), then from (1.3), we can easily see that 


$$
W_{\alpha, \beta}^{k, \delta} f(z)=z+\sum_{n=2}^{\infty}\left[\sum_{m=1}^{k}\left(\begin{array}{l}
k \\
m
\end{array}\right)(-1)^{m+1}\left(\frac{\alpha^{m}+n \beta^{m}}{\alpha^{m}+\beta^{m}}\right)\right]^{\delta} a_{n} z^{n} .
$$

Remark 1.1. It should be remarked that the operator $W_{\alpha, \beta}^{k, \delta}$ generalizes some known operators considered earlier

(1) For $k=1$, the operator $W_{\alpha, \beta}^{1, \delta}=I_{\alpha, \beta}^{\delta}$ was introduced and studied by Swamy [8].

(2) For $\beta=1$ and $\alpha>-1$, the operator $W_{\alpha, 1}^{1, \delta}=I_{\alpha}^{\delta}$ was investigated by Cho and Kim [2] and Cho and Srivastava [3].

(3) For $k=1, \alpha=1-\beta$ and $\beta \geq 0$, the operator $W_{1-\beta, \beta}^{1, \delta}=D_{\beta}^{\delta}$ was given by AlOboudi [1].

(4) For $k=1, \alpha=0$ and $\beta=1$, the operator $W_{0,1}^{1, \delta}=S^{\delta}$ was considered by Sălăgean [7].

We will require the following definition and lemmas in proving our present investigation.

Definition 1.2 [5]. Let $Q$ be the family of all functions that are holomorphic and injective on $\overline{\mathbb{D}} \backslash \mathcal{E}(q)$, where

$$
\mathcal{E}(q)=\left\{r \in \partial \mathbb{D}: \lim _{z \rightarrow r} q(z)=\infty\right\},
$$

and $q^{\prime}(r) \neq 0$ for $r \in \partial \mathbb{D} \backslash \mathcal{E}(q)$. The subfamily of $Q$ with $q(0)=a$ is denoted by $Q(a)$.

Lemma 1.1 [5]. Suppose that $h$ is holomorphic and convex univalent function in $\mathbb{D}$, $h(0)=a, \lambda \neq 0$ and $\operatorname{Re}(\lambda) \geq 0$. If $p \in \mathcal{H}[a, n]$ and

$$
p(z)+\frac{1}{\lambda} z p^{\prime}(z) \prec h(z),
$$

then

$$
p(z) \prec q(z) \prec h(z),
$$

where $q(z)=\lambda z^{-\lambda} \int_{0}^{z} h(t) t^{\lambda-1} d t$ is convex and it is the best dominant of (1.5). 
Lemma 1.2 [6]. Suppose that the function $h$ is convex in $\mathbb{D}, h(0)=a, \lambda \neq 0$ and $\operatorname{Re}(\lambda) \geq 0$. If $p \in \mathcal{H}[a, n] \cap Q, p(z)+\frac{1}{\lambda} z p^{\prime}(z)$ is univalent in $\mathbb{D}$ and

$$
h(z) \prec p(z)+\frac{1}{\lambda} z p^{\prime}(z),
$$

then

$$
q(z) \prec p(z)
$$

where $q(z)=\lambda z^{-\lambda} \int_{0}^{z} h(t) t^{\lambda-1} d t$ is convex and it is the best subordinant of (1.6).

\section{Key Lemma and Main Results}

Before stating and proving our main results, we first establish the following identities involving the operator defined by (1.4):

Lemma 2.1. Assume that $f \in \mathcal{A}, \alpha \in \mathbb{R}, \beta>0, \alpha+\beta>0, \delta \in \mathbb{N}_{0}=\mathbb{N} \cup\{0\}$ and the operator $W_{\alpha, \beta}^{k, \delta}$ is defined by (1.4). Then

$$
z\left(W_{\alpha, \beta}^{k, \delta} f(z)\right)^{\prime} \cong \Upsilon(\alpha, \beta, m, k) W_{\alpha, \beta}^{k, \delta+1} f(z)-\Phi(\alpha, \beta, m, k) W_{\alpha, \beta}^{k, \delta} f(z)
$$

where $\Upsilon(\alpha, \beta, m, k)$ and $\Phi(\alpha, \beta, m, k)$ have the following binomial series representations:

$$
\Upsilon(\alpha, \beta, m, k)=\sum_{m=1}^{k}\left(\begin{array}{l}
k \\
m
\end{array}\right)(-1)^{m+1}\left(\left(\frac{\alpha}{\beta}\right)^{m}+1\right)
$$

and

$$
\Phi(\alpha, \beta, m, k)=\sum_{m=1}^{k}\left(\begin{array}{l}
k \\
m
\end{array}\right)(-1)^{m+1}\left(\frac{\alpha}{\beta}\right)^{m} .
$$

Proof. In the light of (1.4), we find that

$$
\Upsilon(\alpha, \beta, m, k) W_{\alpha, \beta}^{k, \delta+1} f(z)-\Phi(\alpha, \beta, m, k) W_{\alpha, \beta}^{k, \delta} f(z)
$$




$$
\begin{aligned}
= & \sum_{m=1}^{k}\left(\begin{array}{c}
k \\
m
\end{array}\right)(-1)^{m+1}\left(\left(\frac{\alpha}{\beta}\right)^{m}+1\right) W_{\alpha, \beta}^{k, \delta+1} f(z)-\sum_{m=1}^{k}\left(\begin{array}{l}
k \\
m
\end{array}\right)(-1)^{m+1}\left(\frac{\alpha}{\beta}\right)^{m} W_{\alpha, \beta}^{k, \delta} f(z) \\
= & z+\sum_{n=2}^{\infty} \sum_{m=1}^{k}\left(\begin{array}{l}
k \\
m
\end{array}\right)(-1)^{m+1}\left(\left(\frac{\alpha}{\beta}\right)^{m}+1\right)\left[\sum_{m=1}^{k}\left(\begin{array}{l}
k \\
m
\end{array}\right)(-1)^{m+1}\left(\frac{\alpha^{m}+n \beta^{m}}{\alpha^{m}+\beta^{m}}\right)\right]^{\delta+1} a_{n} z^{n} \\
& -\sum_{n=2}^{\infty} \sum_{m=1}^{k}\left(\begin{array}{c}
k \\
m
\end{array}\right)(-1)^{m+1}\left(\frac{\alpha}{\beta}\right)^{m}\left[\sum_{m=1}^{k}\left(\begin{array}{l}
k \\
m
\end{array}\right)(-1)^{m+1}\left(\frac{\alpha^{m}+n \beta^{m}}{\alpha^{m}+\beta^{m}}\right)\right]^{\delta} a_{n} z^{n} \\
= & z+\sum_{n=2}^{\infty}\left[\left(\sum_{m=1}^{k}\left(\begin{array}{l}
k \\
m
\end{array}\right)(-1)^{m+1}\left(\left(\frac{\alpha}{\beta}\right)^{m}+1\right)\right)\left(\sum_{m=1}^{k}\left(\begin{array}{l}
k \\
m
\end{array}\right)(-1)^{m+1}\left(\frac{\alpha^{m}+n \beta^{m}}{\alpha^{m}+\beta^{m}}\right)\right)\right. \\
& \left.-\sum_{m=1}^{k}\left(\begin{array}{l}
k \\
m
\end{array}\right)(-1)^{m+1}\left(\frac{\alpha}{\beta}\right)^{m}\right]\left[\sum_{m=1}^{k}\left(\begin{array}{l}
k \\
m
\end{array}\right)(-1)^{m+1}\left(\frac{\alpha^{m}+n \beta^{m}}{\alpha^{m}+\beta^{m}}\right)\right]^{\delta} a_{n} z^{n} \\
= & z+\sum_{n=2}^{\infty} n\left[\sum_{m=1}^{k}\left(\begin{array}{l}
k \\
m
\end{array}\right)(-1)^{m+1}\left(\frac{\alpha^{m}+n \beta^{m}}{\alpha^{m}+\beta^{m}}\right)\right]^{\delta} a_{n} z^{n-1} \\
= & z\left(W_{\alpha, \beta}^{k, \delta} f(z)\right)^{\prime},
\end{aligned}
$$

which establishes the identity (2.1).

Definition 2.1. Assume that $\psi$ is an holomorphic and convex univalent function in $\mathbb{D}$ with $\psi(0)=1$ and $\alpha \in \mathbb{R}, \eta, \beta>0, \quad \alpha+\beta>0, \quad \delta \in \mathbb{N}_{0}=\mathbb{N} \cup\{0\}$. A function $f \in \mathcal{A}$ is called in the family $E(\eta, \alpha, \beta, \delta, k, m ; \psi)$ if the following differential subordination is satisfied:

$$
\frac{1}{z}\left[(1-\eta \Upsilon(\alpha, \beta, m, k)) W_{\alpha, \beta}^{k, \delta} f(z)+\eta \Upsilon(\alpha, \beta, m, k) W_{\alpha, \beta}^{k, \delta+1} f(z)\right] \prec \psi(z) .
$$

A function $f \in \mathcal{A}$ is called in the family $F(\eta, \alpha, \beta, \delta, k, m ; \psi)$ if the following differential superordination is satisfied:

$$
\psi(z) \prec \frac{1}{z}\left[(1-\eta \Upsilon(\alpha, \beta, m, k)) W_{\alpha, \beta}^{k, \delta} f(z)+\eta \Upsilon(\alpha, \beta, m, k) W_{\alpha, \beta}^{k, \delta+1} f(z)\right],
$$

where $\Upsilon(\alpha, \beta, m, k)$ is given by (2.2). 
Theorem 2.1. Suppose that $\psi$ is a convex function in $\mathbb{D}$ with $\psi(0)=1$ and $\eta>0$. If $f \in E(\eta, \alpha, \beta, \delta, k, m ; \psi)$, then there is a convex function $q$ such that $q(z) \prec \psi(z)$ and $f \in E(0, \alpha, \beta, \delta, k, m ; q)$.

Proof. Assume that

$$
p(z)=\frac{W_{\alpha, \beta}^{k, \delta} f(z)}{z}=1+\sum_{n=2}^{\infty}\left[\sum_{m=1}^{k}\left(\begin{array}{l}
k \\
m
\end{array}\right)(-1)^{m+1}\left(\frac{\alpha^{m}+n \beta^{m}}{\alpha^{m}+\beta^{m}}\right)\right]^{\delta} a_{n} z^{n-1} .
$$

It is obvious that $p \in \mathcal{H}[1,1]$.

Since $f \in E(\eta, \alpha, \beta, \delta, k, m ; \psi)$, we find that

$$
\frac{1}{z}\left[(1-\eta \Upsilon(\alpha, \beta, m, k)) W_{\alpha, \beta}^{k, \delta} f(z)+\eta \Upsilon(\alpha, \beta, m, k) W_{\alpha, \beta}^{k, \delta+1} f(z)\right] \prec \psi(z) .
$$

Now from (2.1), (2.4) and (2.5), it is evident that

$$
\begin{aligned}
& \frac{1}{z}\left[(1-\eta \Upsilon(\alpha, \beta, m, k)) W_{\alpha, \beta}^{k, \delta} f(z)+\eta \Upsilon(\alpha, \beta, m, k) W_{\alpha, \beta}^{k, \delta+1} f(z)\right] \\
= & p(z)+\eta z p^{\prime}(z) \prec \psi(z) .
\end{aligned}
$$

By making use of Lemma 1.1 with $\lambda=\frac{1}{\eta}$, we deduce that

$$
p(z) \prec q(z) \prec \psi(z) .
$$

It follows from (2.4) that

$$
\frac{W_{\alpha, \beta}^{k, \delta} f(z)}{z} \prec q(z) \prec \psi(z),
$$

where

$$
q(z)=\frac{1}{\eta} z^{-\frac{1}{\eta}} \int_{0}^{z} \psi(t) t^{\frac{1}{\eta}-1} d t
$$

This shows that $f \in E(0, \alpha, \beta, \delta, k, m ; q)$ and the proof is completed.

Theorem 2.2. Suppose that $\psi$ is a convex function in $\mathbb{D}$, with $\psi(0)=1$ and $\eta>0$. 
If $f \in F(\eta, \alpha, \beta, \delta, k, m ; \psi), \frac{W_{\alpha, \beta}^{k, \delta} f(z)}{z} \in \mathcal{H}[1,1] \cap Q$ and

$$
\frac{1}{z}\left[(1-\eta \Upsilon(\alpha, \beta, m, k)) W_{\alpha, \beta}^{k, \delta} f(z)+\eta \Upsilon(\alpha, \beta, m, k) W_{\alpha, \beta}^{k, \delta+1} f(z)\right]
$$

is univalent in $\mathbb{D}$, then there is a convex function $q$ such that $f \in$ $F(0, \alpha, \beta, \delta, k, m ; q)$.

Proof. Assume that the function $p$ is defined by (2.4). It is evident that $p \in \mathcal{H}[1,1] \cap Q$. By routine computations and considering $f \in F(\eta, \alpha, \beta, \delta, k, m ; \psi)$, we conclude that

$$
\psi(z) \prec p(z)+\eta z p_{z}^{\prime}(z)
$$

By making use of Lemma 1.2 with $\lambda=\frac{1}{\eta}$, we obtain

$$
q(z) \prec p(z) \text {. }
$$

In view of (2.4), yields

$$
q(z) \prec \frac{W_{\alpha, \beta}^{k, \delta} f(z)}{z},
$$

where

$$
q(z)=\frac{1}{\eta} z^{-\frac{1}{\eta}} \int_{0}^{z} \psi(t) t^{\frac{1}{\eta}-1} d t
$$

If we combine the results of Theorem 2.1 and Theorem 2.2, we obtain "sandwich theorem".

Theorem 2.3. Assume that $\psi_{1}$ and $\psi_{2}$ are two convex functions in $\mathbb{D}$ with $\psi_{1}(0)$ $=\psi_{2}(0)=1$ and $\eta>0$. If $f \in E\left(\eta, \alpha, \beta, \delta ; k, m ; \psi_{1}\right) \cap F\left(\eta, \alpha, \beta, \delta, k, m ; \psi_{2}\right)$, $\frac{W_{\alpha, \beta}^{k, \delta} f(z)}{z} \in \mathcal{H}[1,1] \cap Q$ and

$$
\frac{1}{z}\left[(1-\eta \Upsilon(\alpha, \beta, m, k)) W_{\alpha, \beta}^{k, \delta} f(z)+\eta \Upsilon(\alpha, \beta, m, k) W_{\alpha, \beta}^{k, \delta+1} f(z)\right]
$$


is univalent in $\mathbb{D}$, then

$$
f \in E\left(0, \alpha, \beta, \delta, k, m ; q_{1}\right) \cap F\left(0, \alpha, \beta, \delta, k, m ; q_{2}\right),
$$

where

$$
q_{1}(z)=\frac{1}{\eta} z^{-\frac{1}{\eta}} \int_{0}^{z} \psi_{1}(t) t^{\frac{1}{\eta}-1} d t
$$

and

$$
q_{2}(z)=\frac{1}{\eta} z^{-\frac{1}{\eta}} \int_{0}^{z} \psi_{2}(t) t^{\frac{1}{\eta}-1} d t
$$

The functions $q_{1}$ and $q_{2}$ are convex.

Theorem 2.4. Suppose that $\psi$ is a convex function in $\mathbb{D}$ with $\psi(0)=1$ and

$$
T(z)=\frac{\tau+2}{z^{\tau+1}} \int_{0}^{z} t^{\tau} f(t) d t, \quad(z \in \mathbb{D}, \operatorname{Re}(\tau)>-2) .
$$

If $f \in E(1, \alpha, \beta, \delta, k, m ; \psi)$, then there is a convex function $q$ such that $q(z) \prec \psi(z)$ and $T \in E(1, \alpha, \beta, \delta, k, m ; q)$.

Proof. Let we choose $p$ by

$$
p(z)=\left(W_{\alpha, \beta}^{k, \delta} T(z)\right)^{\prime}, \quad(z \in \mathbb{D}) .
$$

Equation (2.7) shows that $p \in \mathcal{H}[1,1]$. Consider (2.6), we deduce that

$$
z^{\tau+1} T(z)=(\tau+2) \int_{0}^{z} t^{\tau} f(t) d t
$$

By routine calculations of (2.8), we find that

$$
(\tau+2) f(z)=(\tau+1) T(z)+z T^{\prime}(z)
$$

and

$$
(\tau+2) W_{\alpha, \beta}^{k, \delta} f(z)=(\tau+1) W_{\alpha, \beta}^{k, \delta} T(z)+z\left(W_{\alpha, \beta}^{k, \delta} T(z)\right)^{\prime}
$$


Differentiating the last relation with respect to $z$, we have

$$
\left(W_{\alpha, \beta}^{k, \delta} f(z)\right)^{\prime}=\left(W_{\alpha, \beta}^{k, \delta} T(z)\right)^{\prime}+\frac{z}{\tau+2}\left(W_{\alpha, \beta}^{k, \delta} T(z)\right)^{\prime \prime} .
$$

According to $f \in E(1, \alpha, \beta, \delta, k, m ; \psi)$, we can write

$$
\frac{1}{z}\left[\Upsilon(\alpha, \beta, m, k) W_{\alpha, \beta}^{k, \delta+1} f(z)-\Phi(\alpha, \beta, m, k) W_{\alpha, \beta}^{k, \delta} f(z)\right] \prec \psi(z),
$$

where $\Upsilon(\alpha, \beta, m, k)$ and $\Phi(\alpha, \beta, m, k)$ have the forms (2.2) and (2.3), respectively.

Implementing (2.1) in (2.10), gives us

$$
\left(W_{\alpha, \beta}^{k, \delta} f(z)\right)^{\prime} \prec \psi(z) .
$$

In the light of (2.9) and (2.11), we deduce that

$$
\left(W_{\alpha, \beta}^{k, \delta} T(z)\right)^{\prime}+\frac{z}{\tau+2}\left(W_{\alpha, \beta}^{k, \delta} T(z)\right)^{\prime \prime} \prec \psi(z) .
$$

Replacing (2.7) in (2.12), we can easily obtain that

$$
p(z)+\frac{1}{\tau+2} z p^{\prime}(z) \prec \psi(z) .
$$

Applying Lemma 1.1 with $\lambda=\tau+2$, leads us to

$$
p(z) \prec q(z) \prec \psi(z) .
$$

By virtue of (2.7), yields

$$
\left(W_{\alpha, \beta}^{k, \delta^{\prime}} T(z)\right)^{\prime} \prec q(z) \prec \psi(z),
$$

where

$$
q(z)=(\tau+2) z^{-(\tau+2)} \int_{0}^{z} \psi(t) t^{\tau+1} d t
$$

Theorem 2.5. Suppose that $\psi$ is a convex function in $\mathbb{D}$ with $\psi(0)=1$ and $T(z)$ is defined by (2.6). If $f \in F(1, \alpha, \beta, \delta, k, m ; \psi), \quad\left(W_{\alpha, \beta}^{k, \delta} T(z)\right)^{\prime} \in \mathcal{H}[1,1] \cap Q \quad$ and $\frac{1}{z}\left[\Upsilon(\alpha, \beta, m, k) W_{\alpha, \beta}^{k, \delta+1} f(z)-\Phi(\alpha, \beta, m, k) W_{\alpha, \beta}^{k, \delta} f(z)\right]$ is univalent in $\mathbb{D}$, then there exists a convex function $q$ such that $T \in F(1, \alpha, \beta, \delta, k, m ; q)$. 
Proof. Let the function $p$ be defined by (2.7). It is evident that $p \in \mathcal{H}[1,1] \cap Q$. By routine computations and considering $f \in F(1, \alpha, \beta, \delta, k, m ; \psi)$, we conclude that

$$
\psi(z) \prec p(z)+\frac{1}{\tau+2} z p^{\prime}(z)
$$

Applying Lemma 1.2 with $\lambda=\tau+2$, yields $q(z) \prec p(z)$.

From (2.7), we can write

$$
q(z) \prec\left(W_{\alpha, \beta}^{k, \delta} T(z)\right)^{\prime}
$$

where

$$
q(z)=(\tau+2) z^{-(\tau+2)} \int_{0}^{z} \psi(t) t^{\tau+1} d t
$$

Combining results of Theorem 2.4 and Theorem 2.5, we state "sandwich theorem".

Theorem 2.6. Assume that $\psi_{1}$ and $\psi_{2}$ are two convex functions in $\mathbb{D}$ with $\psi_{1}(0)=\psi_{2}(0)=1$ and $T(z)$ is defined by (2.6). If $f \in E\left(1, \alpha, \beta, \delta, k, m ; \psi_{1}\right) \cap$ $F\left(1, \alpha, \beta, \delta, k, m ; \psi_{2}\right), \quad\left(W_{\alpha, \beta}^{k, \delta} T(z)\right)^{\prime} \in \mathcal{H}[1,1] \cap Q$ and $\frac{1}{z}\left[\Upsilon(\alpha, \beta, m, k) W_{\alpha, \beta}^{k, \delta+1} f(z)\right.$ $\left.-\Phi(\alpha, \beta, m, k) W_{\alpha, \beta}^{k, \delta} f(z)\right]$ is univalent in $\mathbb{D}$, then

$$
T \in E\left(1, \alpha, \beta, \delta, k, m ; q_{1}\right) \cap F\left(1, \alpha, \beta, \delta, k, m ; q_{2}\right),
$$

where

$$
q_{1}(z)=(\tau+2) z^{-(\tau+2)} \int_{0}^{z} \psi_{1}(t) t^{\tau+1} d t \text { and } q_{2}(z)=(\tau+2) z^{-(\tau+2)} \int_{0}^{z} \psi_{2}(t) t^{\tau+1} d t
$$

The functions $q_{1}$ and $q_{2}$ are convex.

\section{References}

[1] F. M. Al-Oboudi, On univalent functions defined by a generalized Sălăgean operator, Int. J. Math. Math. Sci. 2004(27) (2004), 1429-1436.

https://doi.org/10.1155/S0161171204108090 
[2] N. E. Cho and T. H. Kim, Multiplier transformations and strongly close-to-convex functions, Bull. Korean Math. Soc. 40(3) (2003), 399-410.

https://doi.org/10.4134/BKMS.2003.40.3.399

[3] N. E. Cho and H. M. Srivastava, Argument estimates of certain analytic functions defined by a class of multiplier transformations, Math. Comput. Modelling 37 (1-2) (2003), 39-49. https://doi.org/10.1016/S0895-7177(03)80004-3

[4] S. S. Miller and P. T. Mocanu, Second order differential inequalities in the complex plane, J. Math. Anal. Appl. 65 (1978), 289-305. https://doi.org/10.1016/0022-247X(78)90181-6

[5] S. S. Miller and P. T. Mocanu, Differential Subordinations. Theory and Applications, Series on Monographs and Textbooks in Pure and Applied Mathematics, Vol. 225, Marcel Dekker Inc., New York, Basel, 2000.

[6] S. S. Miller and P. T. Mocanu, Subordinants of differential superordinations, Complex Variables 48(10) (2003), 815-826. https://doi.org/10.1080/02781070310001599322

[7] G. St. Sălăgean, Subclasses of univalent functions, Lecture Notes in Math., Springer Verlag, Berlin 1013 (1983), 362-372. https://doi.org/10.1007/BFb0066543

[8] S. R. Swamy, Inclusion properties of certain subclasses of analytic functions, Int. Math. Forum 7(36) (2012), 1751-1760. 